\title{
Numerical Optimal Control of a Spherical Parallel Manipulator Based on Unique Kinematic Solutions
}

\author{
Almas Shintemirov, Member, IEEE, Aibek Niyetkaliyev and Matteo Rubagotti, Member, IEEE
}

\begin{abstract}
This paper presents a framework for generating optimal motor trajectories for a spherical parallel manipulator (SPM) with revolute joints, actuated by servomotors with default internal position control settings. The proposed framework consists of three phases. First, an approach to obtain unique forward kinematics is introduced, in order to relate the angular positions of the servomotors to the orientation of the SPM top mobile platform. Then, a configuration space for the SPM is defined by using numerical procedures, in order to guarantee the absence of singularities and of collisions between links during the motion of the manipulator. Finally, reference trajectories of the servomotors are defined via convex optimization. These trajectories determine an optimal evolution of the SPM motion, based on configuration space and original servomotor dynamics. The proposed strategy is experimentally demonstrated on a prototype of Agile Wrist SPM with three servomotors.
\end{abstract}

Index Terms-Spherical parallel manipulators, robot kinematics, motion planning, optimal control, optimization methods.

\section{INTRODUCTION}

Parallel mechanisms are widely used in applications requiring speed, precision and rigidity with limited workspace, e.g. in medical and industrial robotics, flight and automobile simulators, mechatronic systems [1]-[5]. Among numerous types of parallel manipulators, spherical parallel manipulators (SPMs) can be applied for designing orientation wrist platforms for industrial robot end effectors, solar or parabolic antenna orientation systems, medical and rehabilitation robots [6]-[9]. A special case of a three degree-of-freedom (3-DOF) SPM with revolute joints (RRR type) named Agile Eye is proposed in [10] for designing a novel camera orientation system. A modification of the Agile Eye, the Agile Wrist, with enhanced load-carrying capacity and reduced weight, is extensively studied in [11]-[15]. Nevertheless, trajectory generation and application development of 3-DOF RRR type SPMs are still challenging tasks, due to the closed kinematic structure of the manipulators, that limits the motion of the robot top mobile platforms and creates complex kinematic singularities in their workspace [16]. These challenges presently limit widespread practical application of SPMs, despite their promising industrial potential as orientating devices.

This work was partially supported by the Ministry of Science and Education of Kazakhstan within target research funding scheme (grant agreement 1042015).

A. Shintemirov is with the Department of Robotics and Mechatronics, Nazarbayev University, 53 Kabanbay Batyr Ave, Astana 010000 Kazakhstan.

A. Niyetkaliyev is with National Laboratory Astana and the Department of Robotics and Mechatronics, Nazarbayev University, 53 Kabanbay Batyr Ave, Astana 010000 Kazakhstan.

M. Rubagotti is with the Department of Engineering, University of Leicester, University Rd, Leicester LE1 7RH, United Kingdom.

Corresponding author: M. Rubagotti. Email: rubagotti @ i eee. org.
Many approaches have been considered to analyze different SPM systems [6]-[20]. In [6] it is shown that the forward kinematic problem of a general 3-DOF RRR SPM leads to a polynomial with at most eight solutions, corresponding to different poses of the manipulator top mobile platform, for a given set of control inputs. However, only one, i.e., unique, kinematic solution, corresponding to the actual physical pose of a parallel manipulator, has to be used for designing a robot control system [21], [22]. A unique kinematic solution of a parallel manipulator can be directly identified using numerical procedures. As an alternative, additional sensors can be used [21], [23], which however will bring extra costs, and the need of dealing with measurement errors [24].

Considering special SPM configurations, e.g. the Agile Wrist/Eye, the complexity of forward and inverse kinematic problems reduces significantly and closed-form analytical solutions can be obtained [19]. An approach for producing a unique solution to the forward kinematics of the Agile Eye SPM is reported in [25]. A similar approach has been applied to analyze the forward kinematics of a specific linearly actuated quadratic SPM in [26].

Workspace analysis of parallel manipulators is a key element for manipulator trajectory planning and control. The workspace of parallel manipulators, in addition to being inherently limited as compared to serial manipulators, is subject to further restrictions due to possible collisions of the links with one another. A number of different methods have been adopted for workspace analysis of parallel manipulators [16], [27]-[32]. According to [32], the SPM assembly modes (as will be defined in Section II) should be taken into account for workspace definition. However, most of the analyses of SPM mechanics do not take the presence of mechanical interference (i.e., collisions) into account [33], [34], which is instead an important aspect in practical applications.

Optimizing the robot motion is important in many applications. The application of optimal control to parallel manipulators has received little attention so far. In particular, optimal trajectory planning problems for a given geometrical path are formulated in [35] for high-speed cable-based parallel manipulators, and in [36] for a Delta parallel robot manipulator. Minimum-time trajectory planning and real-time control of a special five-bar parallel robot is presented in [37]. Even though low-level PID control has been applied to different types of SPMs (see, e.g., [9], [38]), to the best of our knowledge no results have been reported on the application of numerical optimal control methods to these systems.

This paper presents a novel framework for generating optimal actuator trajectories for an SPM system with revolute 
joints, actuated by servomotors with default internal position control settings. The proposed strategy consists of three phases. At first, the kinematic analysis of 3-DOF SPMs with revolute joints is revisited in Section II and an approach for obtaining unique solutions to forward kinematics is introduced in Section III, in order to univocally relate the angular positions of the SPM actuated joints to the orientation of its top mobile platform. Based on these results, the second phase, outlined in Section IV, focuses on singularity analysis and determination of possible mechanical interference between the SPM links. As a result, the SPM workspace (i.e., the set of all accessible configurations of the manipulator) is defined numerically. The last phase is presented in Section $\mathrm{V}$ and consists in defining reference signals for the SPM actuators, based on workspace and original servomotor dynamics, that generate an optimal evolution of the system dynamics (e.g. minimum-velocity-norm or minimum-time). This is different from simply generating optimal references, in that the optimization is carried out with respect to the actual system trajectory (imposed by its dynamics) for the given reference, rather than considering the reference itself. Due to the need of solving a numerical optimization problem that can easily have hundreds of variables and constraints, the planning of these trajectories requires offline computation, and can therefore be applied only if the initial and final SPM configurations are known a priori. In the proposed framework, the resulting optimization problem is convex (or quasi-convex) [39]: as a consequence, the problem related to the possible convergence to local minima, present in nonlinear optimization problems (cf. [40]), is overcome. Experimental demonstration of the proposed framework using a 3D printed prototype of the Agile Wrist SPM with three servomotors is given in Section VI, which also includes a discussion of the results. The first phase of the framework proposed in this paper is a development of the authors' preliminary work [41], that also reported an approach for obtaining a unique inverse kinematic solution, a graphical verification method using SolidWorks CAD software and numerical examples considering a specific Agile Wrist SPM.

\section{Fundamental Concepts}

Kinematic analysis of SPMs is a well-developed topic, see e.g. [6]-[20], [25], [42]. In order to help the reader understand the contributions of this paper, a brief summary of the 3-DOF RRR SPM kinematics is introduced in the remainder of this section.

\section{A. Coordinate System}

A model of a general symmetric 3-DOF SPM with revolute joints is shown in Fig. 1. The SPM consists of two pyramidshape platforms: a base and a top mobile platform. These are connected by three equally-spaced legs, numbered by $i=1,2,3$, each of them composed of two curved links, namely proximal (lower) and distal (upper) links. The axes of all joints, denoted by unit vectors $\mathbf{u}_{i}, \mathbf{v}_{i}$, and $\mathbf{w}_{i}$, intersect at a common point, referred to as center of rotation. The dimensions of proximal and distal links are denoted as $\alpha_{1}$ and

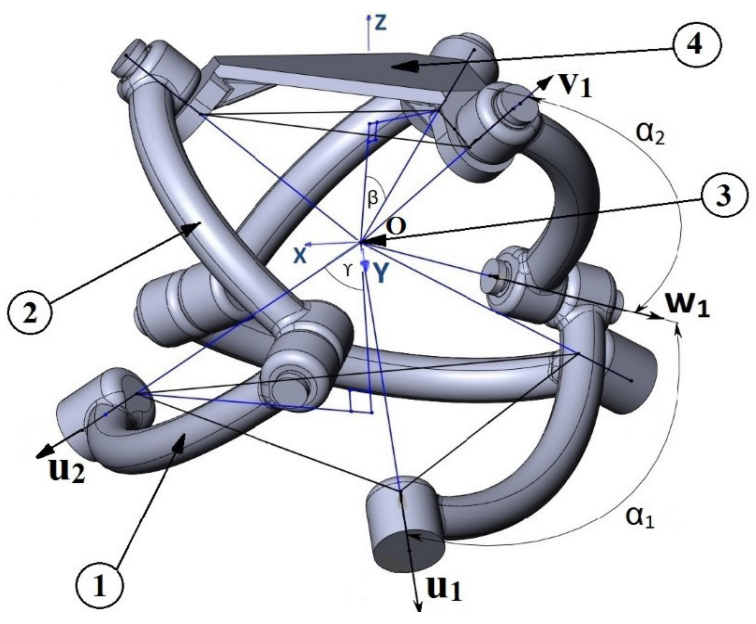

Fig. 1. 3D representation of the kinematic model of a 3-DOF RRR SPM: (1) - proximal link, (2) - distal link, (3) - center of rotation, (4) - top mobile platform.

$\alpha_{2}$, respectively. Angles $\beta$ and $\gamma$ define the geometry of two regular pyramids of the top mobile and base platforms. The motion of the top mobile platform is confined on the surface of a sphere centered at the center of rotation.

The right-handed orthogonal coordinate system with its origin located at the SPM center of rotation is shown in Fig. 1. The $z$ axis is normal to the base pyramid platform and is directed upwards, while the $y$ axis is located in the plane generated by the $z$ axis and $\mathbf{u}_{1}$. The input joint angles, which can be imposed by using three electrical servomotors, are referred to as $\theta_{i}, i=1,2,3$, and measured from the plane generated by the $z$ axis and $\mathbf{u}_{i}$, to the plane of a proximal link.

The unit vectors $\mathbf{u}_{i}, i=1,2,3$, of the base joint axes are defined as follows [12]:

$$
\mathbf{u}_{i}=\left[\begin{array}{lll}
\sin \eta_{i} \sin \gamma, & \cos \eta_{i} \sin \gamma, \quad-\cos \gamma
\end{array}\right]^{T},
$$

where $\eta_{i}, i=1,2,3$, are the angles measured from the plane generated by the $z$ axis and $u_{1}$, to the plane generated by the $z$ axis and $u_{i}$. By symmetry, $\eta_{i}=2(i-1) \pi / 3$ [7].

Unit vectors $\mathbf{w}_{i}, i=1,2,3$, correspond to the axes of the SPM intermediate revolute joints and are obtained in terms of input joint angles $\theta_{i}$ [12]:

$$
\mathbf{w}_{i}=\left[\begin{array}{c}
s \eta_{i} s \gamma c \alpha_{1}-\left(c \eta_{i} s \theta_{i}-s \eta_{i} c \gamma c \theta_{i}\right) s \alpha_{1} \\
c \eta_{i} s \gamma c \alpha_{1}+\left(s \eta_{i} s \theta_{i}+c \eta_{i} c \gamma c \theta_{i}\right) s \alpha_{1} \\
-c \gamma c \alpha_{1}+s \gamma c \theta_{i} s \alpha_{1}
\end{array}\right],
$$

where $s$ and $c$ denote sine and cosine, respectively.

Unit vectors $\mathbf{v}_{i}, i=1,2,3$, that are parallel to the axes of SPM top revolute joints, define orientation of the SPM top mobile platform.

\section{B. SPM Kinematics}

Considering the SPM forward kinematics, both $\mathbf{u}_{i}$ and $\mathbf{w}_{i}$, $i=1,2,3$, are assumed to be known. Vectors $\mathbf{w}_{i}$ are defined as functions of the actuated joint angles $\theta_{i}$. The task is to find the unknown vectors $\mathbf{v}_{i}, i=1,2,3$, of the SPM top mobile platform [42]. Different approaches to obtain solutions to the SPM forward kinematics problem lead to formulating 
eighth-degree polynomial equations, providing at most eight forward kinematic solutions [6], [12], commonly referred to as assembly modes [18]. In this work, the general SPM analysis approach is adopted, in which $\mathbf{v}_{i}, i=1,2,3$, are treated as unknown unit vectors [12], implying the condition

$$
\left\|\mathbf{v}_{i}\right\|=1
$$

$\|\cdot\|$ indicating the Euclidean norm, that will be directly used in the numerical algorithms described in Section III. For the SPM closed kinematic chain, the following equations hold [12]:

$$
\mathbf{w}_{i} \cdot \mathbf{v}_{i}=\cos \alpha_{2}, \quad i=1,2,3 .
$$

The geometry of the regular pyramid of the SPM top mobile platform implies [12]

$$
\mathbf{v}_{i} \cdot \mathbf{v}_{j}=\cos \alpha_{3}, \quad i, j=1,2,3, \quad i \neq j,
$$

where $\alpha_{3}$ is the angle between the axes of the $i$-th and $j$-th top joints. It is equal to the angle between the lateral edges of the top pyramid, which takes the value [12]

$$
\alpha_{3}=2 \sin ^{-1}\left(\sin \beta \cos \frac{\pi}{6}\right) .
$$

A combination of equations (3)-(5) is utilized to generate a system of three linear and six quadratic equations, which is solved numerically to obtain a unique solution to the SPM forward kinematics as proposed in Section III. Once the unique solution to the SPM forward kinematics is found, the unit vector $\mathbf{n}$, normal to the SPM top mobile platform, can be defined as a function of the corresponding components of vectors $\mathbf{v}_{i}$ as follows:

$$
\mathbf{n}=\frac{\mathbf{N}}{\|\mathbf{N}\|},
$$

where $\mathbf{N}=\mathbf{v}_{1}+\mathbf{v}_{2}+\mathbf{v}_{3}$. The case in which $\mathbf{n}=\left[\begin{array}{lll}0 & 0 & 1\end{array}\right]^{T}$ corresponds to the SPM mobile platform being parallel to the base platform in the coordinate system specified in Section II-A.

If the orientation of the SPM mobile platform is given (i.e., vectors $\mathbf{v}_{i}$ are known) inverse kinematic solutions are defined by three uncoupled equations for actuated joint angles $\theta_{i}$ as presented in [7], [15]. For any given orientation of the SPM top mobile platform, there are two solutions exist for each angle $\theta_{i}$ on each SPM leg $i=1,2,3$ [7], also referred to as working modes [18].

\section{Singularity Analysis}

After defining the the vector of actuated joint angles

$$
\boldsymbol{\theta} \triangleq\left[\begin{array}{lll}
\theta_{1} & \theta_{2} & \theta_{3}
\end{array}\right]^{T}
$$

the Jacobian matrix $\mathbf{J}$ maps the angular velocity vector $\boldsymbol{\omega}$ of the SPM mobile platform into the vector of actuated joint rates $\dot{\boldsymbol{\theta}}$ as follows [7]:

$$
\dot{\boldsymbol{\theta}}=\mathbf{J} \boldsymbol{\omega} .
$$

An equivalent formulation of (9) can be written in terms of matrices $\mathbf{J}_{1}$ and $\mathbf{J}_{2}$ [43]:

$$
\mathbf{J}_{1} \boldsymbol{\omega}+\mathbf{J}_{2} \dot{\boldsymbol{\theta}}=0
$$

in which

$$
\begin{gathered}
\mathbf{J}_{1}=\left[\begin{array}{c}
\left(\mathbf{v}_{1} \times \mathbf{w}_{1}\right)^{T} \\
\left(\mathbf{v}_{2} \times \mathbf{w}_{2}\right)^{T} \\
\left(\mathbf{v}_{3} \times \mathbf{w}_{3}\right)^{T}
\end{array}\right] \\
\mathbf{J}_{2}=\operatorname{diag}\left(\mathbf{u}_{1} \times \mathbf{w}_{1} \cdot \mathbf{v}_{1}, \mathbf{u}_{2} \times \mathbf{w}_{2} \cdot \mathbf{v}_{2}, \mathbf{u}_{3} \times \mathbf{w}_{3} \cdot \mathbf{v}_{3}\right) .
\end{gathered}
$$

The Jacobian is finally obtained as

$$
\mathbf{J}=\mathbf{J}_{1}^{-1} \mathbf{J}_{2}
$$

The first type of singularity occurs when $\operatorname{det}\left(\mathbf{J}_{2}\right)=0$. This corresponds to vectors $\mathbf{u}_{i}, \mathbf{w}_{i}$ and $\mathbf{v}_{i}, i=1,2,3$, lying in the same plane, i.e., the corresponding SPM leg is completely folded or unfolded. The condition for this type of singularity can be obtained from equation (12) as $\left(\mathbf{u}_{i} \times \mathbf{w}_{i}\right) \cdot \mathbf{v}_{i}=0$, $i=1,2,3$ [31].

These singular configurations correspond to the set of SPM poses for which the finite number of different working (assembly) modes changes, thus defining the boundary of an SPM workspace in a particular working mode (assuming no joint limits and no link interference), i.e., [18], [27], [43].

The second type of singularity occurs when $\operatorname{det}\left(\mathbf{J}_{1}\right)=0$. In general, as opposed to the first one, the corresponding configuration may be located inside the workspace [31].

The third kind of singularity occurs when, for certain configurations, both $\mathbf{J}_{1}$ and $\mathbf{J}_{2}$ are singular [43].

The SPM singularity conditions can be detected using the condition number of its Jacobian matrix, defined as [7]:

$$
\kappa=\|\mathbf{J}\|\left\|\mathbf{J}^{-1}\right\|
$$

with

$$
\|\mathbf{J}\|=\sqrt{\operatorname{tr}\left(\mathbf{J}^{T} \mathbf{W} \mathbf{J}\right)} .
$$

Here, matrix $\mathbf{W}$ is defined as

$$
\mathbf{W}=\frac{1}{3} \mathbf{I},
$$

with $\mathbf{J} \in \mathbb{R}^{3 \times 3}$, I denoting the $3 \times 3$ identity matrix.

To simplify numerical computations, the so-called conditioning index is used [7]

$$
\zeta(\mathbf{J})=\frac{1}{\kappa(\mathbf{J})}, \quad 0<\zeta(\mathbf{J})<1 .
$$

A value of $\zeta(\mathbf{J})$ close to zero denotes a singular configuration of the SPM. On the other hand, a value $\zeta(\mathbf{J})$ in the immediate vicinity of 1 corresponds to an isotropic configuration (i.e., a perfectly conditioned Jacobian matrix) and is used to design optimal configurations of SPMs [44].

\section{UNIQUE FORWARD KINEMATICS}

In order to achieve the objective of defining SPM optimal trajectories, the first phase consists in obtaining unique forward kinematic solutions for a given SPM system. In this way, after defining the SPM actuator trajectories in the form of time evolutions of the reference actuator angles $\theta_{i}, i=1,2,3$, one can always obtain the corresponding evolution of the other geometric variables of the system. This will be needed, for instance, for verifying whether a particular configuration leads to a singularity condition of the SPM. Here, the term 
configuration is referred to a particular value for the vector of input joint (i.e., actuator) angles $\boldsymbol{\theta}$. The approach is presented in the remainder of this section in the form of an algorithm for obtaining a unique forward kinematic solution of a general SPM with revolute joints.

A unique solution for the forward kinematics in the form of three unit vectors $\mathbf{v}_{i}, i=1,2,3$, is obtained using Algorithm 1 below.

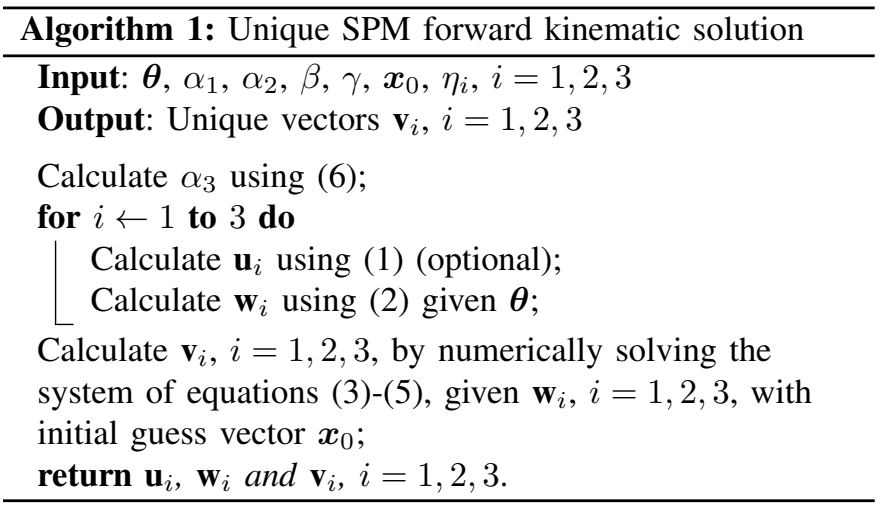

Analyses of the Agile Eye SPM design presented in [18] and [25] prove that the current Agile Eye/Wrist SPM forward kinematic solution lies in the same working mode and assembly mode as the initial (home) assembly mode (initial configuration of the robot) and is reachable through SPM continuous motion without crossing a singularity, i.e., without changing assembly mode. This condition may not hold for an arbitrary SPM with revolute joints which may be cuspidal, in the sense that it may change its assembly mode without crossing a first-type singularity. Since the kinematic equations of parallel manipulators are very complex, the definition of geometric conditions that lead to a cuspidal parallel robot design is still an open research problem [18], [45]. However, utilizing the definition proposed in [22], stating that "noncuspidal parallel manipulators are defined as parallel manipulators with none of their legs, as a serial structure, being cuspidal", we may assume that most of the 3-DOF SPMs with revolute joints are, in fact, noncuspidal manipulators. According to [45], each SPM leg $i=1,2,3$, considered as an RRR series manipulator, is noncuspidal, since the degree of its inverse kinematics polynomial, e.g. as formulated in [7], [15], is no greater than 2 .

Considering an arbitrarily given general 3-DOF SPM design with revolute joints, its initial (home) assembly mode can be chosen employing the assembly analysis presented in [12]. As a result, the most isotropic SPM assembly mode is selected with $\zeta(\mathbf{J})$ being closest to 1 , that may ensure the largest singularity-free workspace. One example of such analysis is presented in [12] for the case of a 3-DOF RRR SPM with coaxial input shafts. Hereafter, we define an SPM home configuration as a non-singular assembly with all proximal links rotated to one side and attached to the base platform through actuated joints, as shown in Fig. 1.

Numerical methods for solving systems of nonlinear algebraic equations require an initial guess, and in most cases converge to the solution that is the closest to the initial guess
[22]. Since we expect to find the current forward kinematic solution that lies in the same assembly mode, the manipulator home position may be used as an initial guess [22] when numerically solving the system of nonlinear equations (3)-(5).

In the present case, the initialization of the numerical method is given by the initial guesses of the $x, y$ and $z$ components of $\mathbf{v}_{i}, i=1,2,3$, gathered in vector $\boldsymbol{x}_{0}$. By changing the signs of the 9 components of $x_{0}$, all possible eight solutions for the SPM forward kinematics, i.e., vectors $\mathbf{v}_{i}$, can be found. The sequence of signs corresponding to one current forward kinematic solution can be selected using the orientation of vectors $\mathbf{v}_{i}$ of an SPM in its home configuration. It can be obtained by projecting unit vectors $\mathbf{v}_{i}$ onto $x, y$ and $z$ axes of the coordinate system. Thus, to obtain numerically the unique solution of the SPM forward kinematics, the signs of $x, y$ and $z$ coordinates of unit vectors $\mathbf{v}_{i}, i=1,2,3$, at the SPM home position are used in the initial guess vector.

Assuming that the same home SPM assembly and coordinate system are considered for obtaining unique solutions to the manipulator kinematics, vector $\boldsymbol{\theta}$ can be found knowing vectors $\mathbf{v}_{i}, i=1,2,3$, corresponding to the current orientation of the SPM top mobile platform, by determining unique inverse kinematic solutions, as proposed by the authors in [41].

\section{WORKSPACE DEFINITION}

The second phase of the proposed framework consists in defining a set of feasible configurations. This section shows how to determine whether a given vector $\boldsymbol{\theta}$ corresponds to an SPM singular configuration (singularity detection), or causes two or more SPM link collisions for that specific configuration (link interference).

\section{A. Singularity Detection}

Utilizing the SPM singularity analysis outlined in Section II-C, for a given vector $\boldsymbol{\theta}$ it is possible to obtain the corresponding vectors $\mathbf{v}_{i}, i=1,2,3$, through forward kinematics. Since $\mathbf{u}_{i}$ and $\mathbf{w}_{i}, i=1,2,3$, are fixed geometrical quantities, it is possible to state that $\mathbf{J}=\mathbf{J}(\boldsymbol{\theta})$. It is then immediate to determine whether a given vector $\boldsymbol{\theta}$ corresponds to SPM singular or near-singular configurations by calculating and comparing the conditioning index $\zeta(\mathbf{J})$ with the minimum threshold value $\zeta(\mathbf{J})_{\min }$, as presented in Algorithm 2.

\section{B. Link Interference}

The determination of possible link interference for a given SPM configuration $\boldsymbol{\theta}$ is not a straightforward task using analytical methods. In the approach proposed in this paper, the SPM links are approximated by using sets of line segments with coordinates defined as functions of $\boldsymbol{\theta}$, knowing the SPM geometry.

Considering the kinematic model of a general SPM with revolute joints (Fig. 1), it can be assumed that each SPM proximal and distal links $i=1,2,3$, can be approximated by line segments that have the SPM joints and the centers of the links as extremes. The coordinates of the end points are defined by the SPM geometry and vectors $\mathbf{u}_{i}, \mathbf{w}_{i}$ and $\mathbf{v}_{i}$ for 


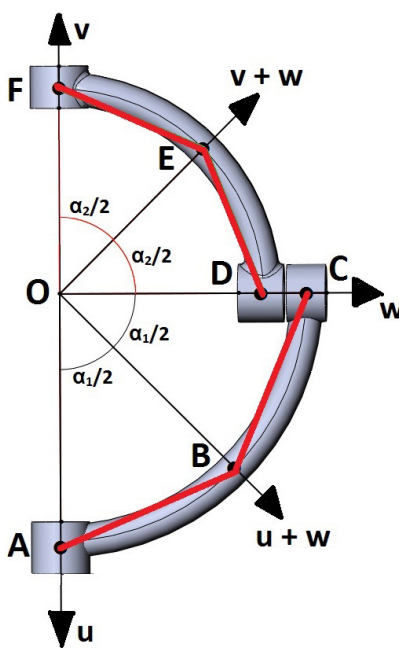

(a)

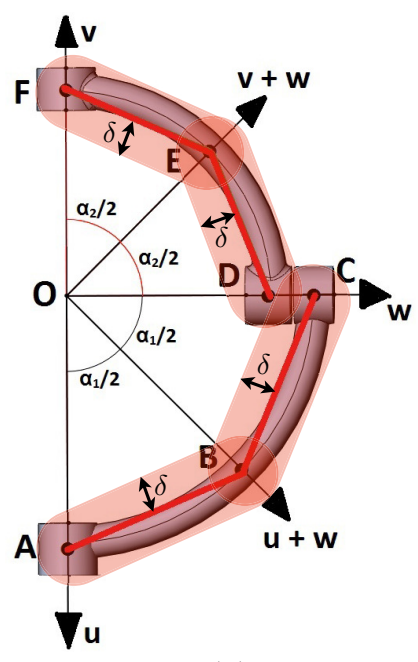

(b)
Fig. 2. Approximation of SPM proximal and distal links by line segments (a), and sets containing all points within a distance $\delta$ from the segments (b)

any given vector $\boldsymbol{\theta}$. Thus, the line segments corresponding to the links are uniquely located in the SPM coordinate systems, and the interference can be determined by calculating the minimum Euclidean distance between any two line segments (the approximation of robot links with segments for interference analysis is already present in the literature, see, e.g., [46]).

Let points $\mathrm{A}_{i}, \mathrm{~B}_{i}, \mathrm{C}_{i}, \mathrm{D}_{i}, \mathrm{E}_{i}$ and $\mathrm{F}_{i}$ denote end points of the line segments approximating proximal and distal links $i=1,2,3$, as shown in Fig. 2. Knowing the SPM geometry and the unit vectors $\mathbf{u}_{i}, \mathbf{w}_{i}$ and $\mathbf{v}_{i}$, the coordinates of points $\mathrm{A}_{i}, \mathrm{~B}_{i}$ and $\mathrm{C}_{i}$ for proximal links $i=1,2,3$, are found as

$$
\begin{aligned}
\mathrm{A}_{i} & =\mathbf{u}_{i} \cdot \mathrm{OA}, \\
\mathrm{B}_{i} & =\frac{\mathbf{u}_{i}+\mathbf{w}_{i}}{\left\|\mathbf{u}_{i}+\mathbf{w}_{i}\right\|} \cdot \mathrm{OB}, \\
\mathrm{C}_{i} & =\mathbf{w}_{i} \cdot \mathrm{OC} .
\end{aligned}
$$

in which $\mathbf{u}_{i}$ and $\mathbf{w}_{i}$ are functions of $\theta_{i}$ as defined by (1) and (2). Similarly, the coordinates of points $\mathrm{D}_{i}, \mathrm{E}_{i}$ and $\mathrm{F}_{i}$ are defined for distal links $i=1,2,3$, as

$$
\begin{aligned}
\mathrm{D}_{i} & =\mathbf{w}_{i} \cdot \mathrm{OD} \\
\mathrm{E}_{i} & =\frac{\mathbf{v}_{i}+\mathbf{w}_{i}}{\left\|\mathbf{v}_{i}+\mathbf{w}_{i}\right\|} \cdot \mathrm{OE}, \\
\mathrm{F}_{i} & =\mathbf{v}_{i} \cdot \mathrm{OF} .
\end{aligned}
$$

Once these points are known for a specific value of $\boldsymbol{\theta}$, the line segments $\mathrm{A}_{i} \mathrm{~B}_{i}, \mathrm{~B}_{i} \mathrm{C}_{i}, \mathrm{D}_{i} \mathrm{E}_{i}$, and $\mathrm{E}_{i} \mathrm{~F}_{i}$ can be determined.

The analysis of a general 3-DOF RRR SPM leg design in Fig. 2a reveals the following observations:

- line segments $A_{i} B_{i}$ cannot collide with segments $B_{i} C_{i}$ of the same distal links;

- line segments $\mathrm{D}_{i} \mathrm{E}_{i}$ cannot collide with segments $\mathrm{E}_{i} \mathrm{~F}_{i}$ of the same proximal links;

- proximal and distal links of the same SPM leg cannot collide with each other, i.e., segments $\mathrm{A}_{i} \mathrm{~B}_{i}$ and $\mathrm{B}_{i} \mathrm{C}_{i}$ never intersect $\mathrm{D}_{i} \mathrm{E}_{i}$ and $\mathrm{E}_{i} \mathrm{~F}_{i}$, for a constant value of $i$.
In order to detect link collision in the cases not specified in the above list, we decided to rely on numerical rather than analytical methods. Each SPM arm is over-approximated in the $3 \mathrm{D}$-space as the union of all points which have a given maximum distance $\delta>0$ from the line segments. In this way, the value of $\delta$ accounts for the thickness of the SPM links, and for the level of approximation that is used to describe each curvilinear link structure with a set of segments. A 2D representation of these sets is reported in Fig. 2b, while a more sophisticated 3D graphical representation is presented for the case study in Section VI-D. For any pair of segments, a sufficient condition for interference is that the minimum Euclidean distance between them be less than $2 \delta$. For any two given segments in the 3D space, this condition can be easily verified by solving a quadratic program (QP). In particular, two generic segments $\mathcal{S}_{1}$ and $\mathcal{S}_{2}$ in the $3 \mathrm{D}$ space can be geometrically represented as the convex hull of their two vertices. The fact that a point belongs to any of the segments can therefore be expressed by a system of linear inequalities. Referring to [39, Sec. 4.4], the minimum distance between $\mathcal{S}_{1}$ and $\mathcal{S}_{2}$ is found by solving

$$
\begin{array}{cc}
\underset{\boldsymbol{\theta}_{1}, \boldsymbol{\theta}_{2}}{\operatorname{minimize}}\left\|\boldsymbol{\theta}_{1}-\boldsymbol{\theta}_{2}\right\| \\
\text { subj. to } & \boldsymbol{\theta}_{1} \in \mathcal{S}_{1}, \\
& \boldsymbol{\theta}_{2} \in \mathcal{S}_{2} .
\end{array}
$$

The optimization variables are the $x, y$, and $z$ coordinates of $\boldsymbol{\theta}_{1}$ and $\boldsymbol{\theta}_{2}$. The problem consists of a quadratic cost function and of linear inequality constraints: it is therefore a small QP, which, being a convex optimization problem, is typically solvable in a few microseconds on a desktop computer.

\section{Space of Feasible Configurations}

Sections IV-A and IV-B introduced numerical procedures to determine if a given configuration $\boldsymbol{\theta}$ corresponds to an SPM singular configuration, or to a collision between SPM links. The procedure can be repeated for a 3D grid of different configurations $\boldsymbol{\theta}$, in which all possible coordinates $\theta_{i}$ of the nodes belong, respectively, to vectors

$$
\boldsymbol{\phi}_{i} \triangleq\left\{\theta_{i, 1}, \theta_{i, 2}, \ldots \theta_{i, M_{i}}\right\}, i=1,2,3
$$

and a uniform sampling is employed for simplicity, i.e., $\Delta \triangleq \theta_{i, j+1}-\theta_{i, j}$ is constant.

Let set $\mathcal{V}$ be defined as the union of all nodes corresponding to feasible configurations $\boldsymbol{\theta}$, i.e., those configurations for which singularities and collisions are avoided. Algorithm 2 details the procedure to determine set $\mathcal{V}$.

A possible approximate space of feasible configurations $\mathcal{P}^{\prime}$ is defined as

$$
\mathcal{P}^{\prime} \triangleq\left(\bigcup_{\boldsymbol{\theta} \in \mathcal{V}} \boldsymbol{\theta} \oplus \mathcal{C}_{\Delta}\right) \ominus \mathcal{C}_{\Delta},
$$

$\mathcal{C}_{\Delta}$ being the cube with side equal to $\Delta$, centered at the origin, $\oplus$ and $\ominus$ indicating the Minkowski sum and Pontryagin difference, respectively. In general, set $\mathcal{P}^{\prime}$ is non convex. Therefore, determining trajectories in $\mathcal{P}^{\prime}$ leading to an optimal 
system performance would require the solution of a nonconvex optimization problem. In order to obtain a convex optimization problem instead, for which we can rely on faster and more reliable solvers, an inner approximation of $\mathcal{P}^{\prime}$ is obtained as a polytope (i.e., a bounded polyhedron), denoted as

$$
\mathcal{P} \triangleq\left\{\boldsymbol{\theta} \in \mathbb{R}^{3}: \boldsymbol{A}_{\boldsymbol{p}} \boldsymbol{\theta} \leq \boldsymbol{b}_{\boldsymbol{p}}\right\} \subseteq \mathcal{P}^{\prime}
$$

where $\boldsymbol{A}_{\boldsymbol{p}} \in \mathbb{R}^{n_{p} \times 3}, \boldsymbol{b}_{\boldsymbol{p}} \in \mathbb{R}^{n_{p}}, n_{p}$ being the number of half spaces, the intersection of which defines the polytope.

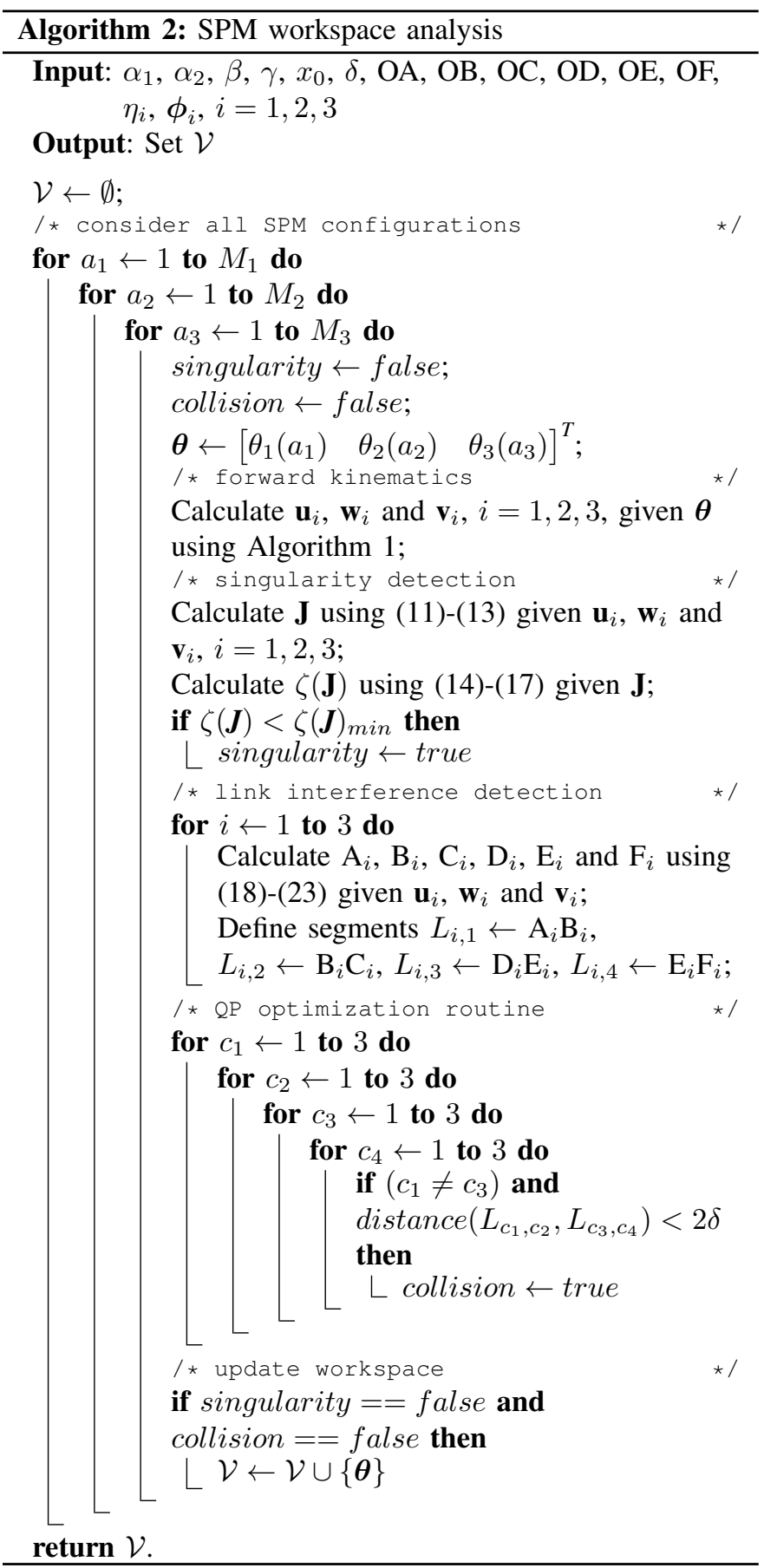

\section{Optimality-Based Trajectory Generation}

Once the space of feasible configurations $\mathcal{P}$ has been formulated as a polytope, it is possible to automatically define feasible trajectories for the SPM motion. In the following, the problem of generating an optimal trajectory of an SPM is considered, given initial and final (desired) configurations of the SPM. In order to formulate an optimal control problem, the SPM dynamics is needed. In many implementations, an SPM is actuated by servomotors with integrated PID controllers implementing internal position control loops. Rather than modifying the default internal servomotor controllers, which is not practical or not possible in many applicative cases, we define suitable reference trajectories that, exploiting the information on the original dynamics of the servomotors, ensure the best possible performance of the SPM system.

An internal control loop of a servomotor is typically tuned so that its closed-loop (linear) dynamics can be represented by a second-order critically-damped system. In state space form it is defined as follows:

$$
\dot{\boldsymbol{x}}_{i}(t)=\left[\begin{array}{cc}
0 & 1 \\
-p^{2} & -2 p
\end{array}\right] \boldsymbol{x}_{i}(t)+\left[\begin{array}{c}
0 \\
p^{2}
\end{array}\right] \theta_{i}^{*}(t), \quad i=1,2,3
$$

where $-p$ is the location of the coinciding real poles of the closed-loop system (assumed to be equal for all SPM actuators), $\boldsymbol{x}_{i} \triangleq\left[\begin{array}{ll}\theta_{i} & \dot{\theta}_{i}\end{array}\right]^{T}$ is the state of $i$-th actuator $(i=1,2,3)$, and $\theta_{i}^{*}$ is the corresponding reference position.

For numerical optimal control, the actuator dynamics (27) can be transformed via exact sampling in discrete-time state space form with sampling interval $T_{s}$, as

$$
\boldsymbol{x}_{i}(k+1)=\boldsymbol{A} \boldsymbol{x}_{i}(k)+\boldsymbol{B} \theta_{i}^{*}(k),
$$

where $k$ is the discrete-time index.

Assume that the initial and final actuator positions (i.e., SPM configuration) $\boldsymbol{\theta}(0), \boldsymbol{\theta}(N) \in \mathbb{R}^{3}$ (corresponding to time instants 0 and $N T_{s}, N$ being a fixed integer number), are fixed and equal to $\boldsymbol{\theta}_{\boldsymbol{d}}(0)$ and $\boldsymbol{\theta}_{\boldsymbol{d}}(N)$, respectively. In addition, the corresponding velocity vectors $\dot{\boldsymbol{\theta}}(0), \dot{\boldsymbol{\theta}}(N) \in \mathbb{R}^{3}$ are equal to zero. Then, the sequence of actuator position references within the considered time window is defined as a single vector

$$
\boldsymbol{\Theta}^{*} \triangleq\left[\begin{array}{lllll}
\boldsymbol{\theta}^{*}(0)^{T} & \boldsymbol{\theta}^{*}(1)^{T} & \ldots & \boldsymbol{\theta}^{*}(N-1)^{T} & \boldsymbol{\theta}^{*}(N)^{T}
\end{array}\right]^{T},
$$

where $\boldsymbol{\theta}^{*}(k) \triangleq\left[\begin{array}{lll}\theta_{1}^{*}(k) & \theta_{2}^{*}(k) & \theta_{3}^{*}(k)\end{array}\right]^{T}$.

\section{A. Minimum-Velocity-Norm Problem}

We first consider the particular problem, known as minimum velocity norm (MVN), of minimizing the Euclidean norm of the servomotors velocity vector during the SPM motion. This type of cost function intrinsically limits the average system speed during the task, ensuring a smooth motion: it has been already proposed for different robotics applications (see, e.g., [47], [48]), but never for parallel manipulators. We formulate 
the MVN problem in our setting as

$$
\begin{array}{cl}
\underset{\boldsymbol{\Theta}^{*}}{\operatorname{minimize}} & \sum_{k=0}^{N} \dot{\boldsymbol{\theta}}(k)^{T} \dot{\boldsymbol{\theta}}(k) \\
\text { subj. to } & \boldsymbol{\theta}(0)=\boldsymbol{\theta}^{*}(0), \boldsymbol{\theta}(N)=\boldsymbol{\theta}^{*}(N) \\
& \dot{\boldsymbol{\theta}}(0)=\dot{\boldsymbol{\theta}}(N)=\mathbf{0} \\
& |\dot{\theta}(k)| \leq \dot{\theta}_{\max }, i=1,2,3, k=0, \ldots, N \\
& \boldsymbol{A}_{\boldsymbol{p}} \theta(k) \leq \boldsymbol{b}_{\boldsymbol{p}}, \quad k=0, \ldots, N \\
& \boldsymbol{x}_{i}(k+1)=\boldsymbol{A x}_{i}(k)+\boldsymbol{B} \theta_{i}^{*}(k), \\
& i=1,2,3, k=0, \ldots, N-1
\end{array}
$$

The equations in (30b) impose the initial and final angular positions of the motors, while (30c) imposes the corresponding angular velocities to be equal to zero. The system of inequalities in (30d) limits the maximum angular velocity of the actuators through the predefined fixed value of $\dot{\theta}_{\max }$. Condition (30e) represents the set of linear inequalities (26), which prevents collisions between links, and singular configurations, during the robot motion. Finally, (30f) imposes the SPM dynamics on the evolution of the actuator angular positions and velocities.

Since (30f) holds, once the initial conditions are defined, the whole SPM motion depends on the sequence of reference values to the manipulator actuators, which is represented by $\Theta^{*}$. The whole system can be represented by six states (vectors $\boldsymbol{x}_{1}$, $\boldsymbol{x}_{2}, \boldsymbol{x}_{3}$ ) and three inputs (vector $\boldsymbol{\theta}^{*}$ ). The cost function can, therefore, be defined as a quadratic function of $\Theta^{*}$, while all conditions in (30b)-(30f) can be represented as linear equality and inequality constraints depending on $\Theta^{*}$ and on the initial condition. This allows one to formulate the problem as a QP, which can be solved very quickly and efficiently, typically relying on active-set or interior-point methods. The convexity of the problem ensures that the optimal sequence of inputs $\Theta^{*}$ is the one leading to the global minimization of the cost function within the given configuration space $\mathcal{P}$.

\section{B. Minimum-Time Problem}

As an alternative to the MVN problem, a minimum-time problem can also be formulated, which consists of finding the smallest positive value of $N$ for which the manipulator can move from $\boldsymbol{\theta}(0)$ to $\boldsymbol{\theta}(N)$ satisfying the same constraints expressed in (30b)-(30f).

$$
\begin{aligned}
\underset{\Theta^{*}}{\operatorname{minimize}} & N \\
\text { subj. to } & (30 \mathrm{~b})-(30 \mathrm{f})
\end{aligned}
$$

This would not be a convex optimization problem, but rather a quasi-convex one, the solution of which can be obtained by iteratively finding a feasible $\Theta^{*}$ satisfying (30b)-(30f) with a different value of $N$ (obtained through a bisection procedure) until the minimum feasible value of $N$ is found (see, for example, [39, Ch. 4]).

Remark 1: Notice that, for both the considered problems, all the constraints are imposed on the actual system variables rather than on their references. Also, the optimization problem is related to the actual variables $\boldsymbol{\theta}$ rather than the reference

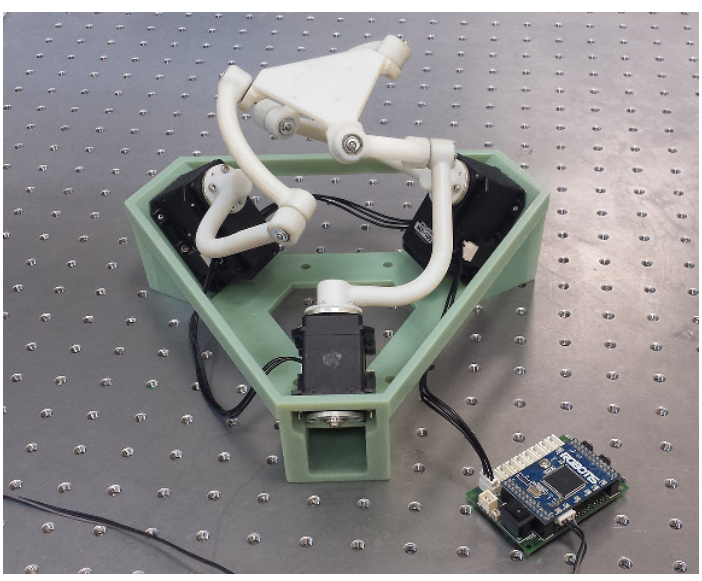

Fig. 3. The experimental setup, consisting of the 3D printed prototype of the Agile Wrist manipulator with the Dynamixel MX-106 servomotors and CM-700 servo controller.

$\boldsymbol{\theta}^{*}$, i.e., there is no need for the references to be perfectly tracked in order to obtain the optimal performance. This means that, for instance, the value of $\boldsymbol{\theta}^{*}$ can exceed the set $\mathcal{P}$ for some time instants, in order to obtain a large error signal $\theta_{i}-\theta_{i}^{*}$ for some of the actuators. This would lead to a fast reaction of the internal actuator controllers, leading in turn to an increase of angular velocity of the SPM actuators. In general, the reference tracking depends on the internal actuator controllers, and cannot be significantly improved. However, using the proposed optimal method for trajectory generation, it is possible to determine the reference that, given the system dynamics, would lead to the optimal evolution of the system variables. It is important to notice that using a wrong system dynamics in (30f) would lead to obtaining reference trajectories that do not optimize the behavior of the actual system. For this reason, it is very important that the closed-loop model of the servomotors, obtained either from physical equations or from system identification, be as precise as possible.

\section{RESULTS AND DisCUSSION}

\section{A. Experimental Setup}

To experimentally demonstrate the application of the proposed framework, a prototype of the "Agile Wrist" SPM has been built. The mechanical part of the prototype has been designed in SolidWorks CAD software and manufactured using 3D printing technology with ABS plastic. The SPM prototype is actuated by three Dynamixel MX-106 servomotors fixed to the SPM base platform and controlled by a CM700 Dynamixel servo controller, as shown in Fig. 3. In the Agile Wrist SPM configuration $\alpha_{1}=\alpha_{2}=90^{\circ}$ (please refer to Section II for the meaning of $\alpha_{1}$ and $\alpha_{2}$ ), with all three legs being identical. The three unit vectors $\mathbf{u}_{i}$ as well as $\mathbf{v}_{i}, i=1,2,3$, are mutually orthogonal [12]. This leads to $\beta=\gamma=54.7^{\circ}$.

The home configuration of the Agile Wrist is chosen such that $\boldsymbol{\theta}=\left[\begin{array}{lll}135^{\circ} & 135^{\circ} & 135^{\circ}\end{array}\right]$ and the axes of base and top joints are aligned as $\mathbf{u}_{1}=-\mathbf{v}_{3}, \mathbf{u}_{2}=-\mathbf{v}_{1}, \mathbf{u}_{3}=-\mathbf{v}_{2}$. In this configuration, the SPM top mobile and base platforms are 
parallel to each other. The angles $\theta_{i}, i=1,2,3$, are set from the vertical plane generated by the $z$ axis and vectors $\mathbf{u}_{i}$, to the plane of the corresponding proximal links in the servomotor counterclockwise direction [41].

\section{B. Calculating SPM Orientation}

1) Forward Kinematic Solution: Consider the case with SPM input angle vector $\boldsymbol{\theta}=\left[\begin{array}{lll}95^{\circ} & 110^{\circ} & 105^{\circ}\end{array}\right]$. The graphical representation of this SPM configuration is shown in [41]. Following Algorithm 1 outlined in Section III all unit vectors $\mathbf{u}_{i}$ and $\mathbf{w}_{i}$ are calculated in MATLAB using (1) and (2). Then, combining equations (3)-(5), the system of three linear and six quadratic equations is generated as follows:

$$
\left\{\begin{array}{l}
-0.9962 v_{1 x}-0.0503 v_{1 y}-0.0712 v_{1 z}=0 \\
0.2989 v_{2 x}+0.9125 v_{2 y}-0.2793 v_{2 z}=0 \\
0.6123 v_{3 x}-0.7618 v_{3 y}-0.2114 v_{3 z}=0 \\
v_{1 x} \cdot v_{2 x}+v_{1 y} \cdot v_{2 y}+v_{1 z} \cdot v_{2 z}=0 \\
v_{1 x} \cdot v_{3 x}+v_{1 y} \cdot v_{3 y}+v_{1 z} \cdot v_{3 z}=0 \\
v_{2 x} \cdot v_{3 x}+v_{2 y} \cdot v_{3 y}+v_{2 z} \cdot v_{3 z}=0 \\
v_{1 x}^{2}+v_{1 y}^{2}+v_{1 z}^{2}=1 \\
v_{2 x}^{2}+v_{2 y}^{2}+v_{2 z}^{2}=1 \\
v_{3 x}^{2}+v_{3 y}^{2}+v_{3 z}^{2}=1
\end{array}\right.
$$

The system of nonlinear equations (32) with 9 unknown components of vectors $\mathbf{v}_{i}$ is solved in MATLAB using function fsolve with the initial guess vector

$$
\boldsymbol{x}_{0}=\left[\begin{array}{lllllllll}
-1 & 1 & 1 & 1 & 1 & 1 & -1 & -1 & 1
\end{array}\right]^{T} .
$$

corresponding to vector

$$
\left[\begin{array}{lllllllll}
v_{1 x} & v_{1 y} & v_{1 z} & v_{2 x} & v_{2 y} & v_{2 z} & v_{3 x} & v_{3 y} & v_{3 z}
\end{array}\right]^{T} .
$$

Function fsolve uses the trust-region-dogleg algorithm [49]. The absolute values in vector $x_{0}$ were chosen arbitrarily, whereas the sequence of signs in vector $\boldsymbol{x}_{0}$ in (33) was adopted from the orientation of vectors $\mathbf{v}_{i}, i=1,2,3$, at the Agile Wrist home configuration and then verified with numerous tests using the SolidWorks CAD software.

As a result, the orientation of the Agile Wrist top mobile platform corresponding to the given actuated joint angles is found in terms of unit vectors $\mathbf{v}_{i}$ :

$$
\mathbf{v}_{1}=\left[\begin{array}{c}
-0.0817 \\
0.8230 \\
0.5621
\end{array}\right], \mathbf{v}_{2}=\left[\begin{array}{c}
0.9039 \\
-0.1768 \\
0.3896
\end{array}\right], \mathbf{v}_{3}=\left[\begin{array}{c}
-0.4204 \\
-0.5401 \\
0.7291
\end{array}\right]
$$

The unit vector $\mathbf{n}$ is defined from equation (7) as

$$
\mathbf{n}=\left[\begin{array}{lll}
0.2321 & 0.0613 & 0.9708
\end{array}\right]^{T} .
$$

The solution (34) for the considered SPM was also verified using the unique inverse kinematic solution and the graphical method presented in [41].
TABLE I

ForWARD KINEMATIC SOLUTIONS OF THE AGILE WRIST/EyE SPM OBTAINED WITH DIFFERENT NUMERICAL METHODS AND APPROACH [12]

\begin{tabular}{l|c|c|c}
\hline \hline $\boldsymbol{\theta}$ & $\mathbf{v}_{1}$ & $\mathbf{v}_{2}$ & $\mathbf{v}_{3}$ \\
\hline \hline \multicolumn{4}{c}{ Trust-region-dogleg algorithm (MATLAB) } \\
\hline 108 & -0.276580 & 0.546672 & -0.790536 \\
60 & 0.127085 & -0.794538 & -0.593566 \\
105 & 0.952551 & 0.264311 & -0.150771 \\
\hline \hline \multicolumn{4}{c}{ Newton's method (MAPLE) } \\
\hline 108 & -0.276605 & 0.546401 & -0.790528 \\
60 & 0.127232 & -0.794559 & -0.593706 \\
105 & 0.952523 & 0.264803 & -0.150258 \\
\hline \hline \multicolumn{4}{|c}{ Solution from Table 5 in [12] } \\
\hline 108 & 0.27657 & -0.54652 & 0.79047 \\
60 & 0.12704 & -0.79457 & -0.59379 \\
105 & 0.95259 & 0.26459 & -0.15034 \\
\hline \hline
\end{tabular}

2) Comparison Analysis: To verify the reliability of the unique forward kinematic solutions, we compare two different numerical methods (i.e., the above-mentioned trust-region method and the Newton's method implemented in Maple). Algorithm 1 was implemented using the two mentioned methods to solve the system of nonlinear equations (3)-(5) for the Agile Wrist SPM configuration $\boldsymbol{\theta}=\left[\begin{array}{lll}108^{\circ} & 60^{\circ} & 105^{\circ}\end{array}\right]$, with initial guess vector $\boldsymbol{x}_{0}$ given in (33). The solutions using both numerical methods are listed in Table I, with one of the eight Agile Wrist SPM forward kinematic solutions computed with the approach reported in [12]. It is clear that the obtained forward kinematic solutions are almost identical, with maximum difference equal to the negligible value of $2 \cdot 10^{-4}$, which only depends on two different numerical tolerances set in the termination criteria of the two algorithms. The coordinate system in [12] is defined as in Fig. 1, with the difference that the $x$ axis is pointing in the opposite direction. Hence, the $x$ components of the third triplet of vectors $\mathbf{v}_{i}$ in Table I have opposite signs. This confirms that the proposed approach allows one to obtain coinciding SPM unique forwards kinematic solutions in terms of unit vectors $\mathbf{v}_{i}$, regardless of the employed numerical method.

The approach proposed in this paper was also compared in [41] with the approach for obtaining a unique solution to forward kinematics of the Agile Eye SPM proposed in [25]. It was shown that using both approaches, a correct unique forward kinematic solution of the considered SPM is obtained.

\section{Analysis of Different SPM Structures}

Consider the example of a different SPM, with coaxial shafts (collinear actuators) [7], [12] that implies $\gamma=0$. The dimensions of the SPM are given as $\alpha_{1}=45 \mathrm{deg}, \alpha_{2}=90$ $\mathrm{deg}$, and $\beta=60 \mathrm{deg}$. Following Algorithm 1, the SPM unique forward kinematic solution is obtained with the two abovementioned numerical methods, for the case with all input shafts angles $\boldsymbol{\theta}=\left[\begin{array}{lll}0^{\circ} & 0^{\circ} & 0^{\circ}\end{array}\right]$. The eight forward kinematic solutions of this SPM structure are also reported in [12]. The comparison of the unique solutions computed numerically with the corresponding solution (out of eight possible) presented 
TABLE II

FORWARD KINEMATIC SOLUTIONS OF AN SPM WITH COAXIAL SHAFTS OBTAINED WITH DIFFERENT NUMERICAL METHODS AND APPROACH [12]

\begin{tabular}{c|c|c|c}
\hline \hline $\boldsymbol{\theta}$ & $\mathbf{v}_{1}$ & $\mathbf{v}_{2}$ & $\mathbf{v}_{3}$ \\
\hline \hline \multicolumn{4}{c}{ Trust-region-dogleg algorithm (MATLAB) } \\
\hline 0 & -0.707080 & -0.079491 & 0.786571 \\
0 & -0.500016 & 0.862360 & -0.362339 \\
0 & 0.500016 & 0.500016 & 0.500016 \\
\hline \hline \multicolumn{4}{c}{ Newton's method (MAPLE) } \\
\hline 0 & -0.707084 & -0.079484 & 0.786569 \\
0 & -0.500016 & 0.862361 & -0.362345 \\
0 & 0.500016 & 0.500016 & 0.500016 \\
\hline \hline \multicolumn{4}{c}{ Solution from Table 3 in [12] } \\
\hline 0 & -0.70711 & -0.079461 & 0.78656 \\
0 & 0.5 & -0.86235 & 0.36233 \\
0 & 0.5 & 0.49999 & 0.50003 \\
\hline \hline
\end{tabular}

in [12] is given in Table II. The $y$ components of the third triplet of vectors $\mathbf{v}_{i}$ have opposite signs due to the left-handed orthogonal coordinate system used in [12]. It can be concluded that the general approach proposed in this paper to obtain the SPM unique forward kinematic solution can be applied to different 3-DOF SPM structures with revolute joints.

\section{Workspace Computation}

As described in Section IV, the workspace of the Agile Wrist top mobile platform is calculated by verifying whether a given SPM configuration vector $\boldsymbol{\theta}$ results in a singular configuration or link interference in the manipulator, as detailed in Algorithm 2. Analysis of the Agile Wrist SPM CAD design [41] and its prototype in Fig. 3 reveals that the parts of the SPM proximal links attached to the actuators, i.e., corresponding to line segment $\mathrm{A}_{i} \mathrm{~B}_{i}$ in Fig. 2, do not collide with other links, and can therefore be neglected. Thus, each proximal link of the Agile Wrist is approximated by a single segment $\mathrm{B}_{i} \mathrm{C}_{i}$ to simplify the link interference analysis. The graphical representation of the line segments approximating the Agile Wrist SPM in its home configuration is shown in Fig. 4.

A set of uniformly-sampled input SPM configurations $\theta$ between $65^{\circ}$ and $155^{\circ}$, with spacing $\Delta=2^{\circ}$ is adopted for the numerical computations, based on preliminary analysis of the Agile Wrist CAD model [41] and experiments with its prototype (Fig. 3). Following Algorithm 2 for all nodes $\boldsymbol{\theta}$ in the grid, the SPM unique forwards kinematic solutions are calculated according to Algorithm 1, as shown in Section VI-B1, based on the geometrical dimensions of the Agile Wrist prototype given in Section VI-A. The obtained unique forwards kinematic solutions are then used to calculate the SPM conditioning index $\zeta(\mathbf{J})$ according to (11)-(17), which is employed for the detection of the singular configurations with threshold $\zeta(\mathbf{J})_{\min }=0.25$.

Subsequently, the coordinates of points $\mathrm{B}_{i}, \mathrm{C}_{i}, \mathrm{D}_{i}, \mathrm{E}_{i}$, and $\mathrm{F}_{i}, i=1,2,3$, are calculated according to (19)-(23). The segments $\mathrm{B}_{i} \mathrm{C}_{i}, \mathrm{D}_{i} \mathrm{E}_{i}, \mathrm{E}_{i} \mathrm{~F}_{i}, i=1,2,3$, are defined in the MATLAB environment using the Multi-Parametric Toolbox
[50], which implements computational geometry functions. This toolbox is also used to easily implement the QP that calculates the Euclidean distance between any two segments by setting $\delta=14 \mathrm{~mm}$, chosen based on the Agile Wrist SPM prototype geometry given in Section IV-B.

A space of feasible configurations $\mathcal{P}$ for the Agile Wrist is obtained in the 3D space defined by $\boldsymbol{\theta}$ by defining $n_{p}=61$ inequalities, is shown in Fig. 5.

\section{E. Trajectory Generation}

1) Actuator Dynamics: The internal PID controllers of the three Agile Wrist SPM actuators receive desired reference positions $\boldsymbol{\theta}^{*}$ as shown in Fig. 6 . The reference values are stored in the memory of the CM-700 servo controller, and are passed to the servomotors with a sampling interval $T_{s}=20 \mathrm{~ms}$. The closed-loop dynamics of the MX-106 servomotors used in the Agile Wrist prototype can be characterized using (27), as explained in Section V, with $p=32.22$, obtained from experimental data via system identification. The discrete equivalent of system (28) is found by using the Tustin method, as follows:

$$
\boldsymbol{A}=\left[\begin{array}{cc}
0.5481 & 0.0435 \\
-45.1937 & 3.3534
\end{array}\right], \quad \boldsymbol{B}=\left[\begin{array}{c}
0.4519 \\
45.1937
\end{array}\right]
$$

2) MVN Optimal Control: An optimal control problem in form (30) is solved, by setting $N=150$, which corresponds to a time interval of $3 \mathrm{~s}$. It was decided not to impose the bound (30d) on the maximum actuator speed reference, which remains implicitly bounded due to the formulation of the cost function. The initial and final SPM configurations are imposed as follows:

$$
\begin{aligned}
\boldsymbol{\theta}^{*}(0) & \triangleq\left[\begin{array}{lll}
127.53^{\circ} & 83.23^{\circ} & 85.25^{\circ}
\end{array}\right]^{T} ; \\
\boldsymbol{\theta}^{*}(N) & \triangleq\left[\begin{array}{lll}
66.80^{\circ} & 155.00^{\circ} & 131.48^{\circ}
\end{array}\right]^{T} .
\end{aligned}
$$

Both vectors lie on the boundary of the set $\mathcal{P}$ in Fig. 5, in order to test the actual absence of collisions between links during experimental trials, which is actually achieved.

The optimal reference sequence $\Theta^{*}$ is obtained by solving the corresponding QP problem (30) using the CVX solver [51] on a MacBook Pro with a $2.9 \mathrm{GHz}$ Intel Core i7 CPU and 8 GB $1600 \mathrm{MHz}$ RAM. The definition and solution of the QP is performed in $25 \mathrm{~s}$.

The obtained position and velocity signals are reported in Figs. 7 and 8. As can be seen from Fig. 7, the measured actuator angular positions are indistinguishable from the simulated ones, which confirms the validity of the identified closed-loop model of the actuating servomotors, and, as a consequence, certifies the optimality of the system evolution also in practice. The velocity signals in Fig. 8 are obtained by using a Kalman filter, which merges the information on the measured position signals with the nominal evolution of the system variables.

3) Minimum-Time Optimal Control: This control problem is formulated according to (31). In this case, a bound on the maximum angular velocities of the Agile Wrist SPM actuators have to be imposed, and is set to $\dot{\theta}_{\max }=50^{\circ} \%$ in (30d). The 


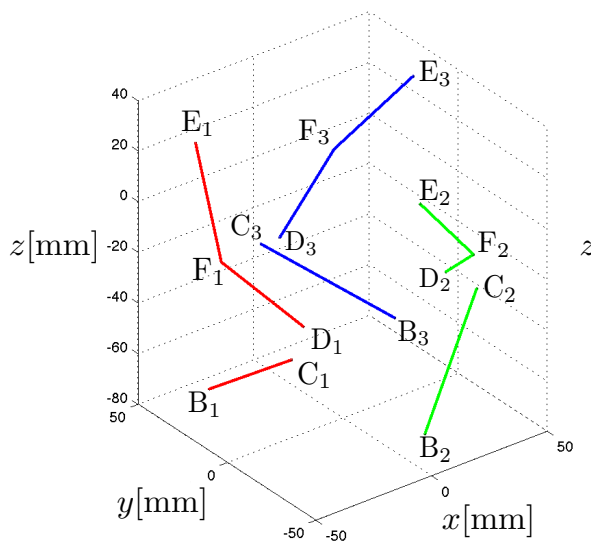

(a)

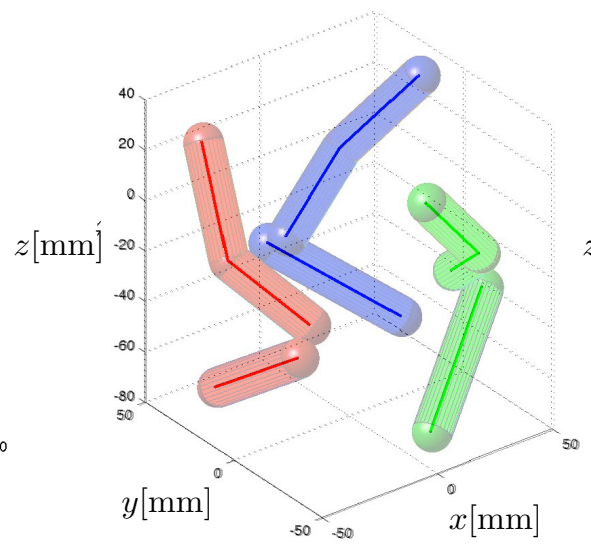

(b)

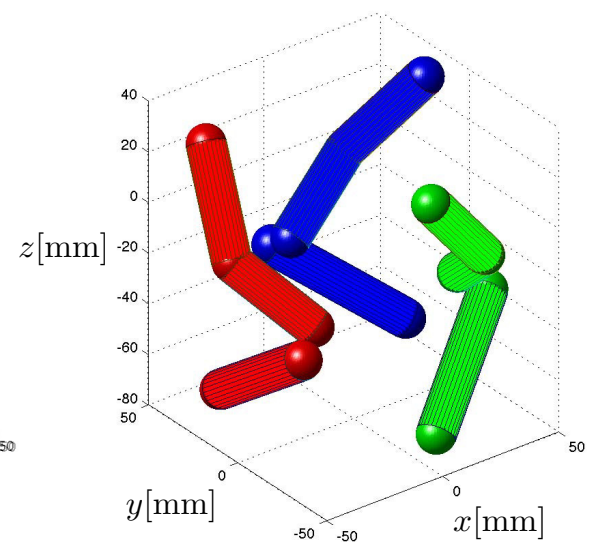

(c)

Fig. 4. Graphical representation of the approximation of the links in the $x-y$ - $z$ space for workspace computation: (a) - segments approximation, (b) cylindrical shapes around the segments, which should not intersect for different arms (i.e., different colors), (c) - direct representation of the cylindrical shapes, which include the mechanical parts of each arm.

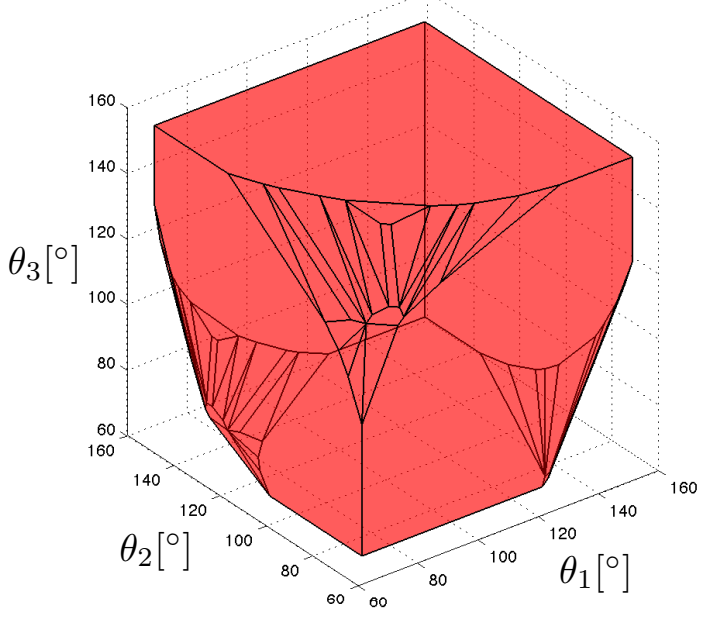

Fig. 5. Graphical representation of the obtained space of feasible configurations $\mathcal{P}$, for the Agile Wrist SPM prototype.

initial and final SPM configurations are set as

$$
\begin{gathered}
\boldsymbol{\theta}^{*}(0) \triangleq\left[\begin{array}{lll}
70.00^{\circ} & 90.00^{\circ} & 80.00^{\circ}
\end{array}\right]^{T} ; \\
\boldsymbol{\theta}^{*}(N) \triangleq\left[\begin{array}{lll}
66.80^{\circ} & 155.00^{\circ} & 131.48^{\circ}
\end{array}\right]^{T} .
\end{gathered}
$$

The optimal reference sequence $\Theta^{*}$ is obtained by solving the corresponding QP problem (30) using the CVX solver on the above-described computer. Initializing the bisection routine with $N=100$, it is necessary to solve 8 QPs (30), which overall lead to the solution of the quasi-convex optimization problem. The overall computation required $85 \mathrm{~s}$ and led to the optimal value $N=76$, corresponding to $1.54 \mathrm{~s}$.

Analogously to the MVN case, the position and velocity signals obtained on the Agile Wrist experimental setup are presented in Figs. 9 and 10. Also in this case, the measured actuator angular positions are indistinguishable from the simulated ones, as shown in Fig. 9.

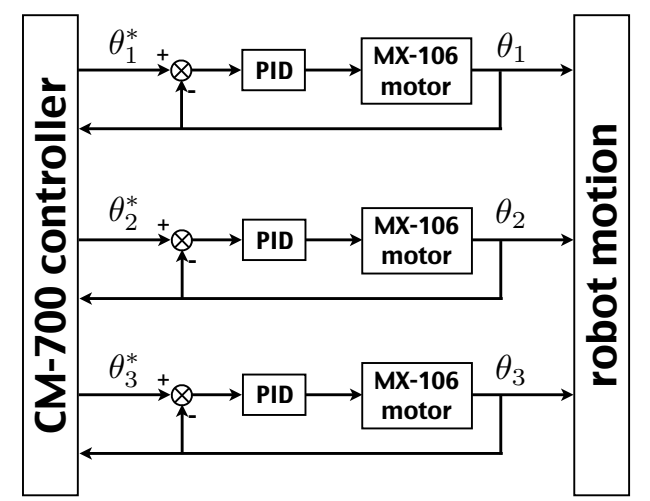

Fig. 6. Conceptual schematic of the implementation of the optimal control scheme.

\section{CONCLUSIONS}

In this paper, a novel framework for the optimal control of a 3-DOF SPM with revolute joints, actuated by servomotors with default internal position control settings, is proposed. The procedure outlined in the framework is composed of three phases: i) determination of an SPM unique forward kinematic solution, ii) numerical construction of a bounded workspace and iii) optimal determination of the actuator references that optimize the SPM motion. The necessary calculations are explained in detail, and presented in the form of pseudocode algorithms suitable for direct computer implementation. An Agile Wrist SPM prototype is built with the purpose of testing the proposed framework. Detailed numerical examples and comparisons demonstrate the application and show the correctness of the proposed approach for identifying SPM unique kinematic solutions. This approach is then employed to define the set of feasible configurations of the Agile Wrist SPM and generate optimal reference trajectories for the servomotors, in order to implement MVN and minimum-time optimal control of the manipulator. The obtained experimental results with the Agile Wrist SPM prototype confirm the feasibility and 

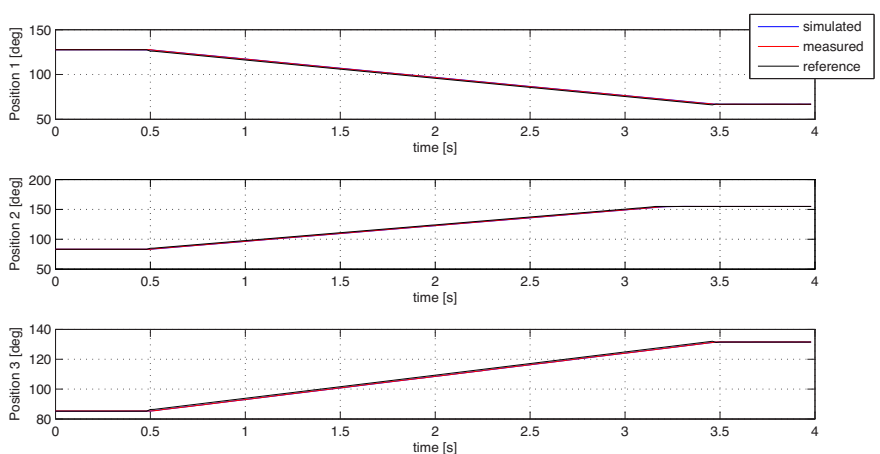

Fig. 7. Experimental results regarding the time evolution of the simulated, measured, and reference angular positions of the servomotor for the MVN problem.

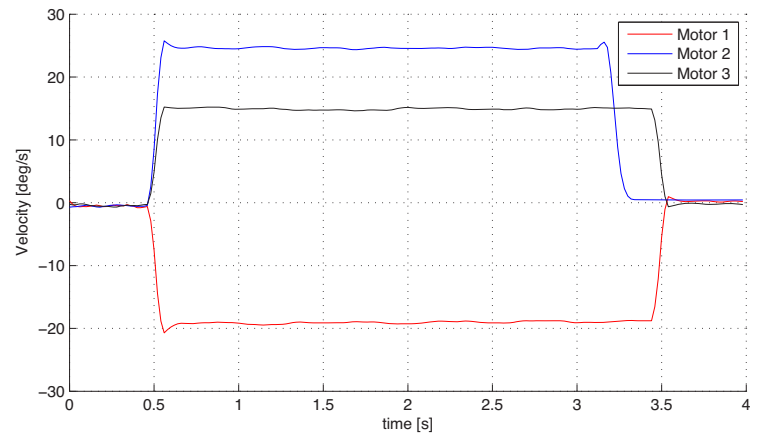

Fig. 8. Time evolution of the estimated angular velocities of the servomotor for the MVN problem.

effectiveness of the proposed framework.

\section{REFERENCES}

[1] M. B. Hong and Y. H. Jo, "Design of a novel 4-DOF wrist-type surgical instrument with enhanced rigidity and dexterity," IEEE/ASME Trans. Mechatronics, vol. 19, no. 2, pp. 500-511, 2014.

[2] J. A. Saglia, N. G. Tsagarakis, J. S. Dai, and D. G. Caldwell, "Control strategies for patient-assisted training using the ankle rehabilitation robot (ARBOT)," IEEE/ASME Trans. Mechatronics, vol. 18, no. 6, pp. 1799 $1808,2013$.

[3] J. Borràs, F. Thomas, and C. Torras, "New geometric approaches to the analysis and design of Stewart-Gough platforms," IEEE/ASME Trans. Mechatronics, vol. 19, no. 2, pp. 445-455, 2014.

[4] F. Pierrot, V. Nabat, S. Krut, and P. Poignet, "Optimal design of a 4-DOF parallel manipulator: From academia to industry," IEEE Trans. Robot., vol. 25, no. 2, pp. 213-224, 2009.

[5] J. Pradipta, M. Klunder, M. Weickgenannt, and O. Sawodny, "Development of a pneumatically driven flight simulator Stewart platform using motion and force control," in Proc. IEEE/ASME Int. Conf. Adv. Int. Mechatronics, 2013, pp. 158-163.

[6] C. Gosselin, J. Sefrioui, and M. J. Richard, "On the direct kinematics of spherical three-degree-of-freedom parallel manipulators of general architecture," ASME J. Mech. Des., vol. 116, no. 2, pp. 594-598, 1994.

[7] C. Gosselin and E. Lavoie, "On the kinematic design of spherical threedegree-of-freedom parallel manipulators," Int. J. Robot. Res., vol. 12, no. 4, pp. 394-402, 1993

[8] T. A. Hess-Coelho, "A redundant parallel spherical mechanism for robotic wrist applications," ASME J. Mech. Des., vol. 129, no. 8, pp. 891-895, 2007.

[9] M. Malosio, S. P. Negri, N. Pedrocchi, F. Vicentini, M. Caimmi, and L. M. Tosatti, "A spherical parallel three degrees-of-freedom robot for ankle-foot neuro-rehabilitation," in Proc. Annual Int. Conf. of the IEEE EMBS, 2012, pp. 3356-3359.

[10] C. Gosselin, E. St-Pierre, and M. Gagni, "On the development of the Agile Eye," IEEE Robot. Automat. Mag., vol. 3, no. 4, pp. 29-37, 1996.
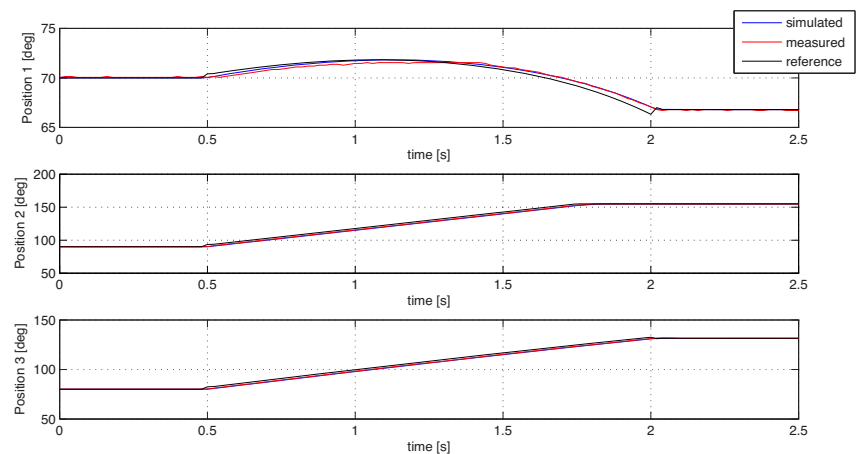

Fig. 9. Experimental results regarding the time evolution of the simulated, measured, and reference angular positions of the servomotor for the minimumtime problem.

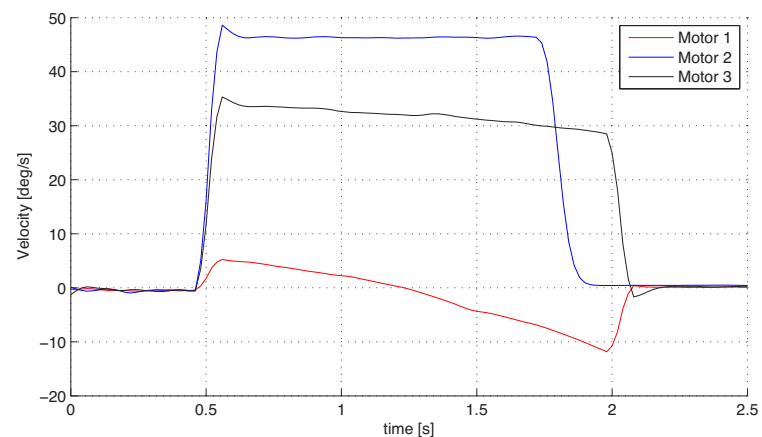

Fig. 10. Time evolution of the estimated angular velocities of the servomotor for the minimum-time problem.

[11] F. Bidault, C. P. Teng, and J. Angeles, "Structural optimization of a spherical parallel manipulator using a two-level approach," in Proc. ASME Design Engineering Tech. Conf., 2001.

[12] S. Bai, M. Hansen, and J. Angeles, "A robust forward-displacement analysis of spherical parallel robots," Mech. Mach. Theory, vol. 44, no. 12, pp. 2204-2216, 2009.

[13] K. Al Widyan, X. Q. Mab, and J. Angeles, "The robust design of parallel spherical robots," Mech. Mach. Theory, vol. 46, no. 3, pp. 335-343, 2011.

[14] S. Staicu, "Recursive modelling in dynamics of Agile Wrist spherical parallel robot," Robot. Cim.-Int. Manuf., vol. 25, no. 2, pp. 409-416, 2009.

[15] Y. Jian and J. Angeles, "Kinematics simulation and control design of the Agile Wrist in a dual-arm robotic mechanical systems," in Proc. Annual Int. Conf. of the IEEE EMBS, 2012, pp. 39-42.

[16] G. Yang, E. Hui, L. Ho, and W. Lin, "A differential geometry approach for the workspace analysis of spherical parallel manipulators," in Proc. World Congress in Mechanism and Machine Science, 2003, pp. 1-6.

[17] S. Bai, M. R. Hansen, and T. O. Andersen, "Modelling of a special class of spherical parallel manipulators with Euler parameters," Robotica, vol. 27 , no. 2, pp. 161-170, 2008

[18] I. A. Bonev, D. Chablat, and P. Wenger, "Working and assembly modes of the Agile Eye," in Proc. IEEE Int. Conf. on Robotics and Automation, Orlando, Florida, 2006, pp. 2317-2322.

[19] C. Gosselin and M. Gagne, "A closed-form solution for the direct kinematics of a special class of spherical three-degree-of-freedom parallel manipulators," in Proc. Workshop Comp. Kinem., 1995, pp. 231-240.

[20] M. C. Palpacelli, L. Carbonari, G. Palmieri, and M. Callegari, "Analysis and design of a reconfigurable 3-DOF parallel manipulator for multimodal tasks," IEEE/ASME Trans. Mechatronics, vol. 20, no. 4, pp. 1975-1985, 2015.

[21] I. A. Bonev, J. Ryu, S. G. Kim, and S. K. Lee, "A closed-form solution to the direct kinematics of nearly general parallel manipulators with optimally located three linear extra sensors," IEEE Trans. Robot. Autom., vol. 17 , no. 2, pp. 148-156, 2001

[22] K. Kamali and A. Akbarzadeh, "A novel method for direct kinematics 
solution of fully parallel manipulators using basic regions theory," Proc. Inst. Mech. Eng. I J. Sys. Cont. Eng., vol. 225, no. 5, pp. 683-701, 2011.

[23] J. P. Merlet, "Closed-form resolution of the direct kinematics of parallel manipulators using extra sensors data," in Proc. IEEE Int. Conf. on Robotics and Automation, 1993, pp. 200-204.

[24] S. F. M. Assal, "Learning multiple solution branches for the direct kinematics of parallel manipulators," in Proc. IEEE Int. Conf. on Mechatronics, 2011, pp. 791-796.

[25] X. Kong and C. Gosselin, "A formula that produces a unique solution to the forward displacement analysis of a quadratic spherical parallel manipulator: the Agile Eye," ASME J. Mech. Robot., vol. 2, no. 4, pp 044 501-1-044 501-4, 2010.

[26] X. Kong, C. Gosselin, and J. M. Ritchie, "Forward displacement analysis of a linearly actuated quadratic spherical parallel manipulator," ASME J. Mech. Robot., vol. 3, no. 1, pp. 011 007-1-011 007-6, 2011.

[27] I. Bonev and C. Gosselin, "Analytical determination of the workspace of symmetrical spherical parallel mechanisms," IEEE Trans. Robot., vol. 22, no. 5, pp. 1011-1017, 2006.

[28] X. J. Liu, J. Wang, and H. Zheng, "Workspace atlases for the computer aided design of the Delta robot," Proc. Inst. Mech. Eng. C J. Mech. Eng. Sci., vol. 217, no. 8, pp. 861-869, 2003.

[29] A. K. Dash, I. M. Chen, S. H. Yeo, and G. Yang, "Workspace generation and planning singularity-free path for parallel manipulators," Mech. Mach. Theory, vol. 40, no. 7, pp. 776-805, 2005.

[30] Y. Li and Q. Xu, "Kinematic analysis and design of a new 3-DOF translational parallel manipulator," ASME J. Mech. Des., vol. 128, no. 4, pp. 729-737, 2006.

[31] C. Gosselin and J. Wang, "Singularity loci of a special class of spherical three-degree-of-freedom parallel mechanisms with revolute actuators," Int. J. Robot. Res., vol. 21, no. 7, pp. 649-659, 2002.

[32] P. Wenger and D. Chablat, "Workspace and assembly modes in fullyparallel manipulators: A descriptive study," in Advances in Robot Kinematics: Analysis and Control. Springer, 1998, pp. 117-126.

[33] J. Hudgens and T. Arai, "Planning link-interference-free trajectories for a parallel link manipulator," in Proc. Int. Conf. on Industrial Electronics, Control, and Instrumentation, 1993, pp. 1506-1511.

[34] Z. Tao and Q. An, "Interference analysis and workspace optimization of 3-RRR spherical parallel mechanism," Mech. Mach. Theory, vol. 69, pp. 62-72, 2013.

[35] S. Behzadipour and A. Khajepour, "Time-optimal trajectory planning in cable-based manipulators," IEEE Trans. Robot., vol. 22, no. 3, pp. 559-663, 2006.

[36] Z. Shareef and A. Trachtler, "Optimal trajectory planning for robotic manipulators using discrete mechanics and optimal control," in Proc. IEEE Multi-conference on Systems and Control, 2014, pp. 240-245.

[37] F. Bourbonnais, P. Bigras, and I. Bonev, "Minimum-time trajectory planning and control of a pick-and-place five-bar parallel robot," IEEE/ASME Trans. Mechatronics, vol. 20, no. 2, pp. 740-749, 2015.

[38] M. Callegari, L. Carbonari, G. Palmieri, N. C. Palpacelli, and D. Tina, "Position control of a 3-CPU spherical parallel manipulator," J. Control Science Eng., vol. 2013, pp. 1-12, 2013.

[39] S. Boyd and L. Vandenberghe, Convex Optimization. Cambridge University Press, 2009.

[40] C. T. Chen and T. T. Liao, "A hybrid strategy for the time-and energyefficient trajectory planning of parallel platform manipulators," Robot. Cim.-Int. Manuf., vol. 27, no. 1, pp. 72-81, 2011.

[41] A. Niyetkaliyev and A. Shintemirov, "An approach for obtaining unique kinematic solutions of a spherical parallel manipulator," in Proc. IEEE/ASME Int. Conf. Adv. Int. Mechatronics, 2014, pp. 1355-1360.

[42] S. Bai and M. R. Hansen, "Forward kinematics of spherical parallel manipulators with revolute joints," in Proc. IEEE/ASME Int. Conf. Adv. Int. Mechatronics, 2008, pp. 532-527.

[43] C. Gosselin and J. Angeles, "Singularity analysis of closed-loop kinematic chains," IEEE Trans. Robot. Autom., vol. 6, no. 3, pp. 281-290, 1990.

[44] _ - "The optimum kinematic design of a spherical three-degree-offreedom parallel manipulator," ASME J. Mech. Des., vol. 111, no. 2, pp. 202-207, 1989.

[45] P. Wenger, "Cuspidal and noncuspidal robot manipulators," Robotica, vol. 25 , no. 6, pp. 677-689, 2007.

[46] J. P. Merlet and D. Daney, "Legs interference checking of parallel robots over a given workspace or trajectory," in Proc. IEEE Int. Conf. on Robotics and Automation, 2006, pp. 757-762.

[47] B. Siciliano and O. Khatib, Springer handbook of robotics. Springer Science \& Business Media, 2008.
[48] Z. Zhang and Y. Zhang, "Variable joint-velocity limits of redundant robot manipulators handled by quadratic programming," IEEE/ASME Trans. Mechatronics, vol. 18, no. 2, pp. 674-686, 2013.

[49] M. J. D. Powell, "A Fortran subroutine for solving systems of nonlinear algebraic equations," in Numerical Methods for Nonlinear Algebraic Equations, P. Rabinowitz, Ed. Gordon \& Breach, 1970, pp. 115-161.

[50] M. Herceg, M. Kvasnica, C. N. Jones, and M. Morari, "Multi-Parametric Toolbox 3.0," in Proc. European Control Conf., 2013, pp. 502-510.

[51] M. Grant, S. Boyd, and Y. Ye, "CVX: Matlab software for disciplined convex programming," 2008. 